\title{
X. Climatologia
}

\section{CARACTERIZAÇÃO FENOLÓGICA DA VIDEIRA 'NIAGARA ROSADA' EM DIFERENTES REGIÕES PAULISTAS $\left({ }^{1}\right)$}

MÁrIO JOSÉ PEDRO JR. $\left({ }^{2,9}\right)$, PAULO CESAR SENTEILIAS $\left({ }^{2}\right)$, CELSO VALDEVINO POMMER $\left.{ }^{3,9}\right)$, FERNANDO PICARELLI MARTINS $\left({ }^{4}\right)$, PAULO BOILER GAILO $\left({ }^{5}\right)$, RUI RIBEIRO DOS SANTOS $\left({ }^{6}\right)$, VIRGINIO BOVI ${ }^{7}$ ) e JOSE C $\triangle R L$ OS SABINO( ${ }^{8}$ )

\section{RESUMO}

Avaliaram-se dados fenológicos da videira 'Niagara Rosada' cm diferentes regiões do Estado de São Paulo para as seguintes épocas de poda: $15 / 7 ; 1 \% / 8 ; 15 / 8$ e $1 \% / 9$, durante os anos agrícolas de 1989/90 c 1990/91, com o objetivo de avaliar o comportamento fenológico $c$ as necessidades tćrmicas da cultura. $\Lambda$ duração das fases fenológicas variou em função do local e da ćpoca de poda. O ciclo total durou de 116 dias para a época de poda $1 \% / 9$, em Tietê c Mococa, até 199 dias para a poda de $15 / 7$ em São Roque. $\Lambda$ temperatura-base para a fase brotação-maturação foi de $12^{\circ} \mathrm{C}$ e a necessidade tćrmica (graus-dia) média, de 1.330 , variando de 1.248 para Jundiaí a 1.386 para São Roque. $\Lambda$ data de poda não influenciou o valor da necessidade térmica num mesmo local.

Termos de indexaçāo: vidcira, Niagara Rosada, fenologia, graus-dia, temperatura-base.

\section{ABSTRACT \\ PHENOLOGICAL CHARACTERIZATION OF NIAGARA ROSADA GRAPE GROWN AT DIFFERENT REGIONS OF THE STATE OF SÃO PAULO (BRAZIL)}

Phenological data of 'Niagara Rosada' grape was evaluated at different localities in the State of São Paulo, for the following prunning dates: July 15; August $1^{\text {st }}$, August 15 and September $1^{\text {st }}$, during the seasons: $1989 / 90$ and $1990 / 91$, with subject of evaluate the phenological behaviour and required total degree-days. The duration of the phenological phases varied as a function of the locality and prunning date and the duration of the total cycle lasted from 116 days for the prunning date

(1) Recebido para publicação em 30 de junho e aceito cm 2 de dezembro de 1993.

( ${ }^{2}$ Sę̧ão de Climatologia $\Lambda$ grícola, Instituto $\Lambda$ gronômico (I $\Lambda$ C), Caixa Postal 28, 13001-970, Campinas (SP).

$\left({ }^{3}\right)$ Seção de Viticultura, I^C.

$\left({ }^{4}\right)$ Estação Experimental de Jundiaí, I $\Lambda \mathrm{C}$.

(5) Estação Experimental de Mococa, I $\wedge \mathrm{C}$.

(5) Estação Experimental de Monte Alegre do Sul, I $\wedge$ C.

$\left({ }^{7}\right)$ Estação Experimental de São Roque, I $\Lambda$ C.

$\left({ }^{8}\right)$ Estação Experimental de Tiètê, I $\Lambda \mathrm{C}$.

( ${ }^{9}$ Com bolsa de pesquisa do CNPq. 
01/09 at Tietê and Mococa, to 199 days for the crop pruned on 15/07 at São Roque. The estimated base-temperature for the period buding/harvest was $12^{\circ} \mathrm{C}$ and the average amount of growing degree-days accumulated was 1,330 with extremes of 1,248 for Jundiai and 1,386 for São Roque. It was noticed that the prunning date did not show influence on the total degree-days needed to complete the period in a same locality.

Index terms: grapes, Niagara rosada, phenology, degree-days, base temperature.

\section{INTRODUÇÃo}

A videira 'Niagara Rosada', amplamente cultivada em São Paulo como uva de mesa, apresenta comportamento diferenciado nas suas diversas regiões ecológicas.

A caracterização fenológica e a quantificação das unidades térmicas necessárias para a vidcira completar as diferentes fases do ciclo vegetativo devem fornecer informações ao viticultor para conhecimento antecipado das prováveis datas de colheita, indicando ainda o potencial climático das regiões para o cultivo e produção de uva.

A videira apresenta uma sucessão de ciclos vegetativos, alternados por períodos de repouso, e locais com climas que não permitcm essa alternância têm limitado seu cultivo (Hidalgo, 1956). O ciclo da videira foi subdividido por Galet (1983) nos seguintes períodos: (a) de crescimento: da brotação ao fim do crescimento; (b) reprodutivo: da floração à maturação; (c) de amadu recimento dos tecidos: da paralisação do crescimento à maturaçao dos ramos; (d) vegetativo: do "choro" à queda das folhas; (c) de repouso: entre dois ciclos vegetativos.

A duração desses períodos é, geralmentc, condicionada pela disponibilidade térmica das regiões de cultivo, tendo a temperatura do ar estreiţa relação com o início da brotação (Pouget, em 1969, citado por Mandelli, 1984) e com a fase de florescimento, influenciando no rendimento da cultura (Carbonneau, 1992, e Turmanidze, 1992).

A caracterização das exigências térmicas da videira mediante 0 conceito de graus-dia tem sido amplamente utilizada (Winkler, 1948; Hidalgo, 1980, Hatch \& Ruiz, 1987, e McIntyre et al., 1987) e, para sua avaliação, são necessárias observações fenológicas em áreas de cultivo situadas $\mathrm{cm}$ diferentes ecossistemas. Tais estudos têm sido feitos por vários autores: Calò (1972) avaliou a fase de florescimento de 140 cultivares, e Forlani et al (1987) caracterizaram a fenologia de dezessete cultivares na Itália.

$\mathrm{Na}$ viticultura brasileira, Passos \& Trintin (1982) tabularam dados dos estádios fenológicos da videira em Bento Gonçalves, e Mandelli (1984), analisando a videira na mesma região, caracterizou a potencialidade climática para seu cultivo pela determinação das necessidades térmicas de diversos cultivares, expressando-as em graus-dia.

O conceito de graus-dia, apesar de suas limitações (McIntyre et al., 1987), tem sido usado para avaliar a duração do ciclo, a produção e a qualidade do produto (Hidalgo, 1980). Além disso, Almcida (1972) considera que as regiões com maiores somas térmicas, em menor espaço de tempo, devem ser indicadas para o cultivo de uva de mesa.

O objetivo deste trabalho, portanto, foi avaliar o comportamento fenológico da videira "Niagara Rosada' em diferentes regiões paulistas, bem como estudar as necessidades térmicas, expressas em graus-dia, para completar as diferentes fases de seu ciclo.

\section{MATERIAL E MÉTODOS}

$\mathrm{O}$ ensaio foi instalado em vinhedos de ' $\mathrm{Nia}$ gara Rosada' nas seguintes Estações Experimentais do Instituto Agronômico: Jundiaí (lat.: $23^{\circ} 12^{\prime} \mathrm{S}$; long.: $46^{\circ} 53^{\prime} \mathrm{W}$; alt.: $715 \mathrm{~m}$ ); Mococa (lat.: $21^{\circ} 28^{\prime} \mathrm{S}$; long.: $47^{\circ} 01^{\prime} \mathrm{W}$; alt: $665 \mathrm{~m}$ ); Tietê (lat.: $23^{\circ} 07^{\prime} \mathrm{S}$; long.: $47^{\circ} 43^{\prime} \mathrm{W}$; alt.: $538 \mathrm{~m}$ ); Monte Alegre do Sul (lat.: $22^{\circ} 41^{\prime} \mathrm{S}$; long.: 
$46^{\circ} 43^{\prime} \mathrm{W}$; alt.: $777 \mathrm{~m}$ ), e São Roque (lat.: $\overline{23}^{\mathrm{\theta}} 32$ 'S; long.: $47^{\circ} 08^{\prime} \mathrm{W}$; alt.: $850 \mathrm{~m}$ ).

Os tratamentos constituíram-se de diferentes épocas de poda: (a) no ano agrícola 1989/90: 15/7, 1\%/8 e 15/8; (b) no ano agrícola 1990/91: $15 / 7,1 \% / 815 / 8$ e $1 \%$. As parcelas tinham três plantas, sendo duas bordaduras e uma central (planta útil), com cinco repetições para cada época de poda.

Efetuaram-se os tratos culturais de acordo com as recomendações técnicas para a cultura, a saber: cobertura morta, tratos fitossanitários, adubações, poda verde e uso de calciocianamida.

A videira foi conduzida sob sistema de espaldeira, com três fios de arame e espaçamento de $2 \times 1 \mathrm{~m}$, exceto na E. E. de Mococa, cujo sistema foi o de manjedoura no espaçamento de $4 \times 2 \mathrm{~m}$. A poda foi do tipo curta, com uma gema em cordão esporonado, c a carga média de gemas por planta, de seis a sete, tendo cada gema, no máximo, dois ramos.

Nas plantas úteis, observaram-se, semanalmente, os estádios fenológicos mediante uma escala de notas variando de 1 a 17 , e que foi adaptada por Pedro Jr. et al, (1990). Nos postos agrometeorológicos das Estações Experimentais, verificaram-se, diariamente, a temperatura mínima e a máxima do ar.

$\mathrm{Na}$ caracterização das exigências térmicas para a videira, utilizou-se o somatório de graus-dia, pelas equações de Villa Nova et al. (1972):

a) $\mathrm{GD}=\left(\mathrm{T}_{\mathrm{m}}-\mathrm{T}_{\mathrm{b}}\right)+\left(\mathrm{T}_{\mathrm{M}}-\mathrm{T}_{\mathrm{m}}\right) / 2$, para $\mathrm{T}_{\mathrm{m}}>\mathrm{T}_{\mathrm{b}}$;

b) $\mathrm{GD}=\left(\mathrm{T}_{\mathrm{M}}-\mathrm{T}_{\mathrm{b}}\right)^{2} / 2\left(\mathrm{~T}_{\mathrm{M}}-\mathrm{T}_{\mathrm{m}}\right)$, para $\mathrm{T}_{\mathrm{m}}<\mathrm{T}_{\mathrm{b}}$;

c) $\mathrm{GD}=0$, para $\mathrm{T}_{\mathrm{b}}>\mathrm{T}_{\mathrm{M}}$,

onde:

GD = graus-dia; $T_{M}=$ temperatura máxima diária $\left({ }^{\circ} \mathrm{C}\right) ; \mathrm{T}_{\mathrm{m}}=$ temperatura mínima diária $\left({ }^{\circ} \mathrm{C}\right)$ e $\mathrm{T}_{b}=$ temperatura-base $\left({ }^{\circ} \mathrm{C}\right)$.

A temperatura-base foi determinada pelo método do menor desvio padrão, como indicado por Arnold (1959).
Os resultados da duração e das necessidades térmicas (graus-dia) das fases fenológicas da videira foram comparados pela análise de intervalos de confiança (Snedecor \& Cochran, 1976).

\section{RESULTADOS E DISCUSSÃO}

\subsection{Duração do ciclo e das fases fenológicas}

As avaliações efetuadas por meio da escala de notas permitiram caracterizar a duração total do ciclo da videira 'Niagara Rosada' e das seguintes fases fenológicas: (a) da poda à brotação: (1-2); (b) da brotação à inflorescência com flores scparadas, início da fase de florescimento: (2-8); (c) da fase anterior à de "chumbinho": (8-12); (d) do "chumbinho" ao grão verde desenvolvido: (12-15); (c) do grão verde à colheita (maturação em torno de $16^{\circ}$ Brix): (15-17). No quadro 1 , encontram-se os valores médios em dias da duração de cada fase fenológica e do ciclo total.

$A$ análise dos intervalos de confiança (Figura 1A) da duração da fase brotação-colheita, nos difcrentes locais e independentemente das épocas de poda, permitiu verificar a seguinte tendência crescente: Mococa, Tietê e Jundiaí (119 a 128 dias); Monte Alegre do Sul (134 dias); São Roque (145 dias). Para o caso da avaliação da influĉncia das épocas de poda (Figura 1B), na duração média da fase, considerada independentemente dos locais, observou-se a seguinte ordem decrescente: $1 \% / 9$ (117 dias); $1 \% / 8$ e $15 / 8$ (130 a 133 dias) e $15 / 7$ (137 dias).

Avaliando a duração do ciclo total (fase: poda-colhcita) nos diferentes locais, em função da ćpoca de poda desde $15 / 7$ a $1 \% / 9$, notou-se a seguinte tendência (Quadro 1): São Roque (149 a 199 dias); Monte Alegre do Sul (132 a 176 dias); Tietê (116 a 164 dias); Jundiaí (130 a 159 dias) e Mococa (116 a 151 dias).

Apesar das diferenças na duração do ciclo total, caracterizou-se uma tendência nas datas de colheita, que se concentraram em períodos de 10 a 15 dias para cada local. Em Mococa, Jundiaí e Tietê, a colheita ocorreu por volta de 23/12 a $1 \% / 1$; cm Monte Alegre do Sul, de 11 a $17 / 1$ e, em São Roque, de $30 / 1$ a $13 / 2$. 


\subsection{Temperatura-base}

Os valores obtidos de duração dos estádios fenológicos, aliados aos de temperatura do ar, permitiram calcular a temperatura-base de desenvolvimento da videira 'Niagara Rosada' pclo método do menor desvio padrão. Na figura 2 são apresentados os resultados obtidos do desvio padrão, em dias, para as diferentes tempcraturas-base.

Para as fases de reprodução $c$ do desenvolvimento da baga (Figuras $2 \mathrm{D}$ c $2 \mathrm{E}$ respectivamente), não foi possivel determinar a tcmperatura-base, provavelmente devido à pequena variabilidade da faixa de temperatura cncontrada durante o transcorrer do experimento.
Para os demais estádios fenológicos, o método utilizado para a determinação da $T_{b}$ (Figuras 2A, B, C c F) detectou os seguintes valores: (a): brotação: $10^{\circ} \mathrm{C}$; (b) desenvolvimento vegetativo: $12^{\circ} \mathrm{C}$; (c) maturação: $14^{\circ} \mathrm{C}$ e (d) brotação-colhcita: $12^{\circ} \mathrm{C}$. Valor idêntico de $\mathrm{T}_{b}$ para a fase brotação-colheita foi obtido por Mandelli (1984) para o cultivar Cabernet Franc em Bento Gonçalves (RS), o qual coincide, também, com o observado por Almcida (1972), que, analisando as possibilidades vitícolas de Angola, constatou ser a $\mathrm{T}_{b}=12^{\circ} \mathrm{C}$ a que melhor se ajustava às nccessidades térmicas da videira. Deve-se ressaltar, porém, que muitos pesquisadores consideram a $\mathrm{T}_{\mathrm{b}}=10^{\circ} \mathrm{C}$ para o ciclo total da videira, incluindo a fase poda-brotação, como Hidalgo (1980) e Turmanidze (1992).

Quadro 1. Duração (dias) do ciclo total c das diversas fases fenológicas para diferentes épocas de poda e locais, no Estado de São Paulo

\begin{tabular}{|c|c|c|c|c|c|c|c|c|}
\hline \multirow{2}{*}{ Local } & \multirow{2}{*}{$\begin{array}{l}\text { Data } \\
\text { da poda }\end{array}$} & \multicolumn{6}{|c|}{ Fase de desenvolvimento } & \multirow{2}{*}{$\begin{array}{l}\text { Ciclo } \\
\text { total }\end{array}$} \\
\hline & & $(1-2)$ & $(2-8)$ & $(8-12)$ & $(12-15)$ & $(15-17)$ & $(2-17)$ & \\
\hline \multirow[t]{4}{*}{ Jundiaí } & $15 / 7$ & 23 & 39 & 19 & 42 & 36 & 136 & 159 \\
\hline & $1 \% / 8$ & 18 & 30 & 22 & 42 & 36 & 130 & 148 \\
\hline & $15 / 8$ & 16 & 28 & 19 & 42 & 40 & 129 & 145 \\
\hline & $1 \% / 9$ & 12 & 26 & 17 & 39 & 36 & 118 & 130 \\
\hline \multirow[t]{4}{*}{ Mococa } & $15 / 7$ & 23 & 21 & 27 & 52 & 28 & 128 & 151 \\
\hline & $1 \% / 8$ & 18 & 20 & 25 & 42 & 32 & 119 & 137 \\
\hline & $15 / 8$ & 14 & 24 & 24 & 39 & 29 & 116 & 130 \\
\hline & $1 \% / 9$ & 4 & 27 & 17 & 32 & 36 & 112 & 116 \\
\hline Monte & $15 / 7$ & 36 & 35 & 27 & 44 & 34 & 140 & 176 \\
\hline Alegre & $1 \% / 8$ & 26 & 33 & 20 & 44 & 43 & 140 & 166 \\
\hline \multirow[t]{2}{*}{ do Sul } & $15 / 8$ & 17 & 31 & 22 & 41 & 41 & 135 & 152 \\
\hline & $1 \% / 9$ & 12 & 29 & 19 & 35 & 37 & 120 & 132 \\
\hline \multirow[t]{4}{*}{ São Roque } & $15 / 7$ & 48 & 37 & 28 & 35 & 51 & 151 & 199 \\
\hline & $1 \% / 8$ & 42 & 35 & 30 & 31 & 54 & 150 & 192 \\
\hline & $15 / 8$ & 31 & 33 & 30 & 32 & 52 & 147 & 178 \\
\hline & $1 \% / 9$ & 19 & 30 & 28 & 33 & 39 & 130 & 149 \\
\hline \multirow[t]{4}{*}{ Tietê } & $15 / 7$ & 34 & 31 & 22 & 41 & 36 & 130 & 164 \\
\hline & $1 \% / 8$ & 20 & 28 & 20 & 42 & 37 & 127 & 147 \\
\hline & $15 / 8$ & 14 & 24 & 21 & 37 & 39 & $12 \mathrm{I}$ & 135 \\
\hline & $1 \% / 9$ & 10 & 23 & 15 & 37 & 31 & 106 & 116 \\
\hline
\end{tabular}



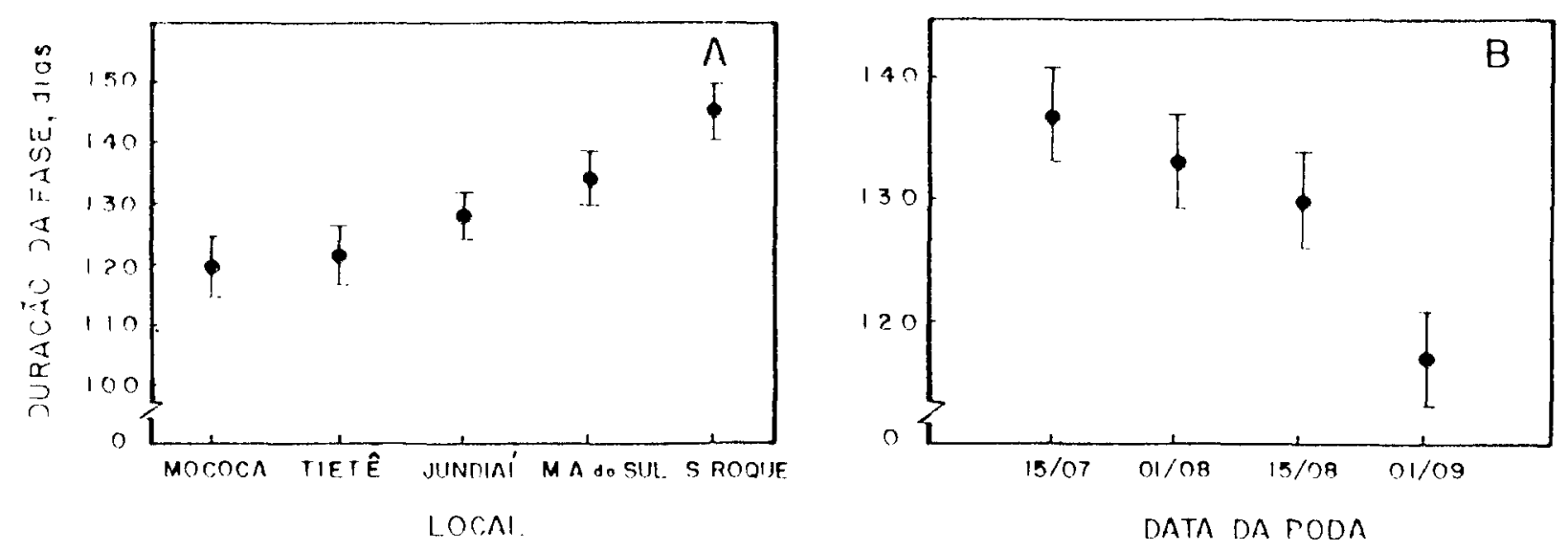

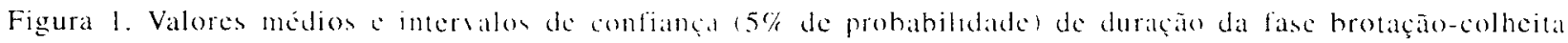
da videira "Niagara Rusda' para diferentes: A: locais e B: Épocis de poda.
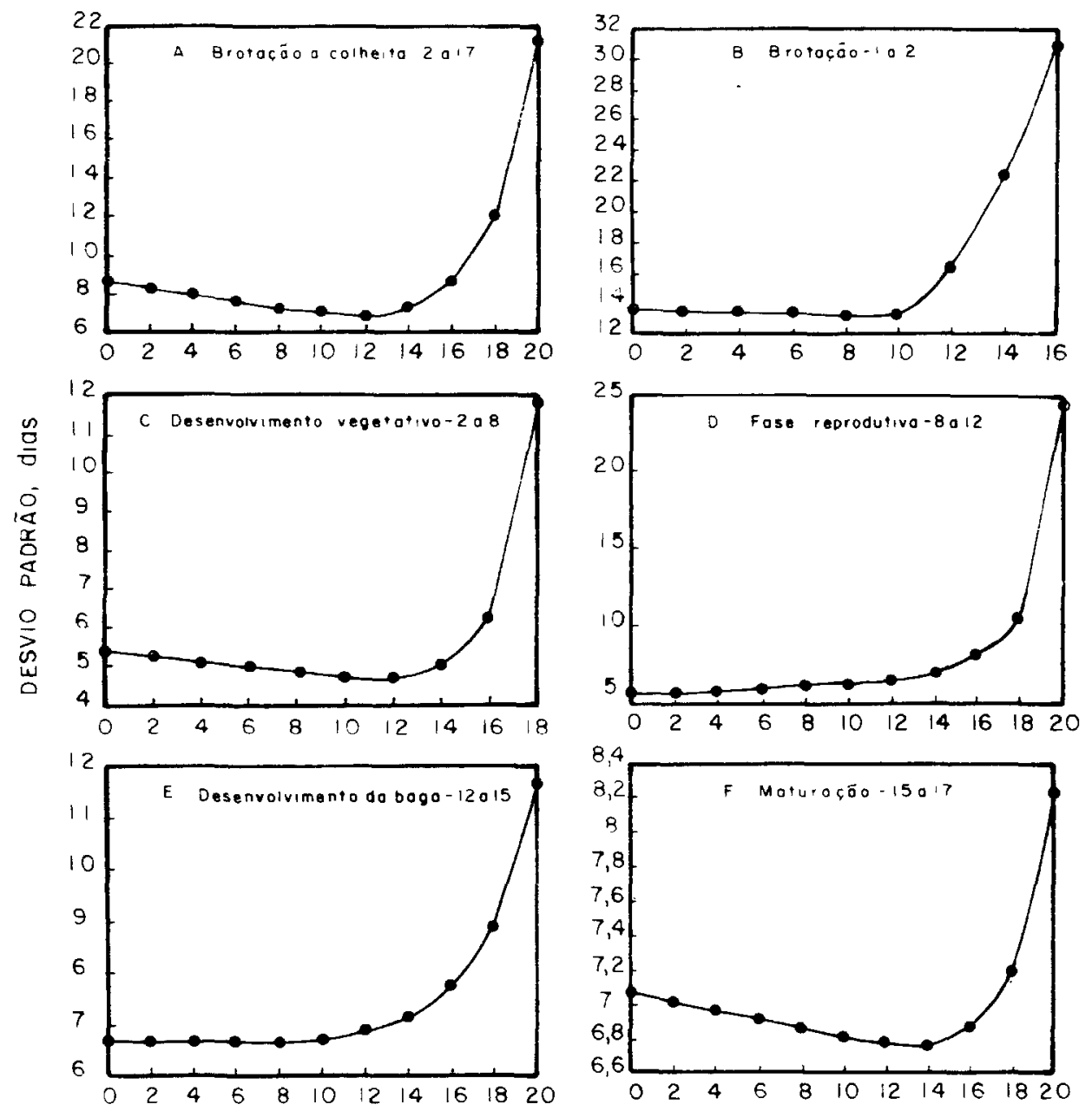

TEMPERATURA BASE, ${ }^{\circ} \mathrm{C}$

Figura 2. Desvio padrão (em dias) da duração das diferentes fases fenológlcas da videira 'Niagara Rosada' $\mathrm{em}$ função da temperatura-base. 


\subsection{Necessidades térmicas (graus-dia).}

Tomando-se como base a $\mathrm{Tb}$ encontrada para cada fase de desenvolvimento, calcularam-se as necessidades térmicas em termos de graus-dia para os locais onde os ensaios foram realizados e para as diferentes épocas de poda (Quadro 2).
Para a fase de desenvolvimento vegetativo (2-8) foram necessários, em média, 280 graus-dia, ressaltando-se que, para as localidades de Jundiaí e Tietê, a época de poda influiu no total de graus-dia no sentido de haver uma diminuição a partir da poda de $15 / 7$ até a de $1 \% / 9$

Quadro 2. Necessidades térmicas (graus-dia) para estádios fenológicos da videira 'Niagara Rosada' para diferentes épocas de poda e locais paulistas

\begin{tabular}{|c|c|c|c|c|c|c|}
\hline \multirow{3}{*}{ Local } & \multicolumn{6}{|c|}{ Fase de Desenvolvimento } \\
\hline & \multirow{2}{*}{$\begin{array}{l}\text { Data } \\
\text { poda }\end{array}$} & $(2-8)$ & $(8-12)$ & $(12-15)$ & $(15-17)$ & \multirow{2}{*}{$\begin{array}{c}(2-17) \\
\mathrm{Tb}=12^{\circ} \mathrm{C}\end{array}$} \\
\hline & & $\mathrm{Tb}=12^{\circ} \mathrm{C}$ & $\mathrm{Tb}=12^{\circ} \mathrm{C}$ & $\mathrm{Tb}=12^{\circ} \mathrm{C}$ & $\mathrm{Tb}=14^{\circ} \mathrm{C}$ & \\
\hline \multirow[t]{5}{*}{ Jundiaí } & $15 / 7$ & 353 & 145 & 390 & 372 & 1.230 \\
\hline & $1 \% / 8$ & 275 & 177 & 415 & 365 & 1.225 \\
\hline & $15 / 8$ & 259 & 166 & 460 & 375 & 1.261 \\
\hline & $1 \% / 9$ & 233 & 186 & 509 & 355 & 1.278 \\
\hline & & & & & Média & $1.248 \mathrm{~d}$ \\
\hline \multirow[t]{5}{*}{ Mococa } & $15 / 7$ & 225 & 257 & 566 & 370 & 1.366 \\
\hline & $1 \% / 8$ & 242 & 252 & 479 & 391 & 1.320 \\
\hline & $15 / 8$ & 270 & 255 & 462 & 372 & 1.286 \\
\hline & $1 \% / 9$ & 302 & 225 & 437 & 413 & 1.362 \\
\hline & & & & & Média & $1.334 \mathrm{abc}$ \\
\hline Monte & $15 / 7$ & 319 & 215 & 505 & 329 & 1.342 \\
\hline Alegre & $1 \% / 8$ & 294 & 181 & 486 & 415 & 1.373 \\
\hline \multirow[t]{3}{*}{ do Sul } & $15 / 8$ & 289 & 201 & 457 & 404 & 1.345 \\
\hline & $1 \% / 9$ & 304 & 200 & 451 & 345 & 1.377 \\
\hline & & & & & Média & $1.359 \mathrm{ab}$ \\
\hline \multirow[t]{5}{*}{ São Roque } & $15 / 7$ & 302 & 231 & 381 & 466 & 1.380 \\
\hline & $1 \% / 8$ & 278 & 288 & 348 & 493 & 1.418 \\
\hline & $15 / 8$ & 295 & 278 & 348 & 482 & 1.415 \\
\hline & $1 \% / 9$ & 325 & 312 & 377 & 332 & 1.329 \\
\hline & & & & & Média & $1.386 \mathrm{a}$ \\
\hline \multirow[t]{5}{*}{ Tietê } & $15 / 7$ & 306 & 183 & 459 & 397 & 1.326 \\
\hline & $1 \% / 8$ & 282 & 172 & 466 & 403 & 1.307 \\
\hline & $15 / 8$ & 233 & 190 & 415 & 440 & 1.279 \\
\hline & $1 \% / 9$ & 225 & 189 & 495 & 345 & 1.243 \\
\hline & & & & & Média & $1.289 \mathrm{bcd}$ \\
\hline \multicolumn{2}{|c|}{ Média geral } & 280 & 215 & 445 & 393 & 1.330 \\
\hline
\end{tabular}

As médias seguidas de mesma letra não diferem entre si. 
Em média, ainda, foram necessários: 215 graus-dia para a fase reprodutiva (8-12); 445 graus-dia para o desenvolvimento da baga (12-15); 393 graus-dia para a maturação (15-17) e 1.330 graus-dia desde a brotação até a colheita (2-17).

O valor necessário de graus-dia (1.330) é bastante semelhante ao obtido por Mandelli (1984), que, trabalhando com dez cultivares de videira vinifera em Bento Gonçalves, obteve resultados na faixa de 1.298 a 1.426 graus-dia. Quando comparados, porém, aos 2.747 graus-dia obtidos por Brink (1974), admite-se que essa superioridade se deveu a estar incluída a fase poda-brotação e ao uso dc $\mathrm{T}_{\mathrm{b}}=10^{\circ} \mathrm{C}$.

A variabilidade encontrada nos resultados fez com que, pela análise do intervalo de confiança, se notasse a influência do local no total de graus-dia necessários para a videira completar a fase brotação-maturação (colheita).

A figura 3 apresenta os valores médios e os intervalos de confiança a $5 \%$ de probabilidade do total de graus-dia (fase 2-17) para diferentes locais e épocas de poda.

O total de graus-dia necessários não diferiu estatisticamente (Figura 3B) quando comparadas as difcrentes épocas de poda, variando entre os extremos de 1.318 para a poda de $1 \% / 9$ a 1.337 para a de $15 / 7$. Entretanto, pode-se observar (Figura 3A) a provável influência do local no total de graus-dia necessários para a fase brotação-colheita, cuja variação foi de 1.248 graus-dia para Jundiaí até 1.386 para São Roque. Esses resultados sugerem que, em regiões de interesse de utilização dos graus-dia para fins de previsão de época de colheita, análises mais detalhadas por local sejam efetuadas.
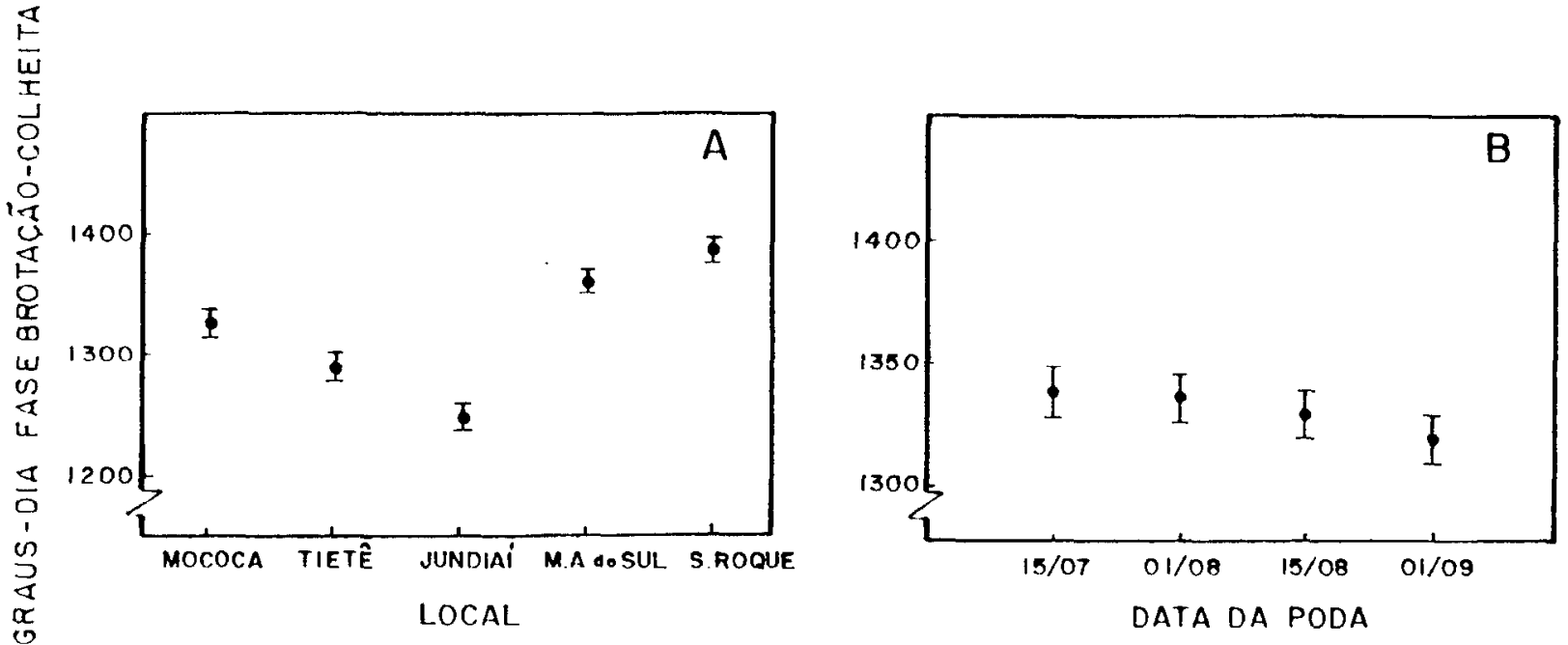

Figura 3. Valores médios e intervalos de confiança (5\% de probabilidade) do total de graus-dia necessários para a fase brotação-colheita da videira 'Niagara Rosada' para diferentes: A: locais e B: épocas de poda.

\section{CONCLUSõeS}

Para a videira 'Niagara Rosada' conduzida em espaldeira, pode-se verificar que:
1. Na brotação-colheita, a temperatura-base foi de $12^{\circ} \mathrm{C}$ e a necessidade térmica para completar a fase, de 1.330 graus-dia, com uma variação cm função do local de 1.248 a 1.386 graus-dia. 
2. A época de poda não influiu no total de graus-dia necessários para completar o ciclo no mesmo local.

\section{REFERÊNCIAS BIBLIOGRÁFICAS}

ALMEIDA, J.L.F. Possibilidades de produção de uvas de mesa em Moçamedes e em Roçadas. Nova Lisboa, Instituto de Investigação Agronômica de $\Lambda$ ngola, 1972. 19p.

ARNOLD, C.Y. The determination and significance of the base temperature in a linear heat unit system. Proceedings of the American Society for Horticultural Science, Geneva, 74:430-445, 1959.

BRINK, C. van den. Predicting harvest date of the 'Concord' grape crop in southwest Michigan. HortScience, Alexandria, 9(3):206-208, 1974.

CALÒ, A. Influenza di alcune condizioni ambientali sull'epoca di fioritura nella Vitis vinifera. Rivista di Viticoltura e di Enologia, Conegliano, 25(4): 149-161, 1972.

CARBONNEAU, A. Meteorologie et viticulture. Geneva, World Meteorological Organization, 1992. 72p. (Rapport CMAg, 42A - WMO/TD 484)

FORLANI, M.; PILONE, N.; COPPOLA, V. \& GIOFFRE, D. Observazioni poliennali sulla fenologia di 17 vitigni e studio delle correlazioni con alcuni indici bioclimatici. Rivista di Viticoltura e di Enologia, Conegliano, 40(11):503-522, 1987.

GALET, P. Précis de viticulture. 4.ed. Montpellier, Déhan, 1983. 584p.

HATCH, R.L. \& RUIZ, M. Influence of pruning date on budbreak of desert table grapes. American Journal of Enology and Viticulture, Lockeford, 38(4):326-328, 1987.

HIDALGO, L. Equivalentes meteorológicos de la vid. Boletin del Instituto Nacional de Investigaciones Agronómicas, Madrid, 16(35):175-209, 1956.

HIDALGO, L. Caracterización macrofisica del ecosistema medio-planta en los viñedos españoles. Madrid, Instituto Nacional de Investigaciones Agrarias, 1980. 255p. (Comunicaciones I.N.I.A. Producción vegetal, 29)
MCINTYRE, G.N.; KLIEWER, W.M. \& RIDER, L.A. Some limitations of the degree day system as used in viticulture in California. American Journal of Enology and Viticulture, Lockeford, 38(2): 128-132, 1987.

M $\Lambda$ NDELLI, F. Comportamento fenológico das principais cultivares de Vitis vinifera $L$. para a região de Bento Gonçalves, RS. Piracicaba, 1984. 125p. Dissertação (Mestrado em Agronomia) - ESALQ-USP, 1984

PASSOS, L.P. \& TRINTIN, P.L. Resposta da videira à variação da época de poda seca: III. Efeitos no comportamento fenológico da Cv. Isabel. Bento Gonçalves, EMBRAPA-UEPAE de Bento Gonçalves, 1982. 6p. (Pesquisa em andamento, 3)

PEDRO JÚNIOR, M.J.; RIBEIRO, I.J.A.; POMMER, C.V. \& MARTINS, F.P. Caracterização de estádios fcnológicos da vidcira Niagara Rosada. In: CONGRESSO BRASILEIRO DE FRUTICULTURA, 10 ., Fortaleza, 1990. Anais. Fortaleza, Sociedade Brasileira de Fruticultura, 1990. v.1, p.453-456.

SNEDECOR, G.W. \& COCIIRAN, W.G. Statistical methods. 6.ed. Ames, Iowa State University Press, 1976. 593p.

TURMANIDZE, T.I. Report on the effect of agrometeorological parameters on viticulture in countries of Eastern Europe. Geneva, World Meteorological Organization, 1992. 48p. (CAgM report, 42B WMO/TD, 500)

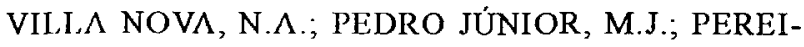
$\mathrm{R} \Lambda$, A.R. \& OMETTO, J.C. Estimativa de grausdia acumulados acima de qualquer temperatura-base, em função das temperaturas máxima e mini$m a$. São Paulo, Universidade de São Paulo - Instituto de Geografia, 1972. 8p. (Caderno de Ciências da Terra, 30)

WINKLER, A.J. Maturity tests for table grapes: the relation of heat summation to time of maturing and palatability. Proceedings of the American Society for Horticultural Science, Geneva, 51:295-298, 1948. 